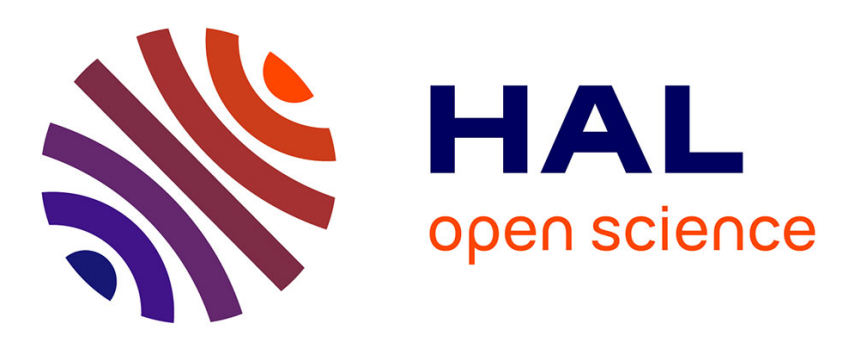

\title{
Universal intensity statistics in a chaotic reverberation chamber to refine the criterion of statistical field uniformity
}

Jean-Baptiste Gros, Ulrich Kuhl, Olivier Legrand, Fabrice Mortessagne, Elodie Richalot

\section{To cite this version:}

Jean-Baptiste Gros, Ulrich Kuhl, Olivier Legrand, Fabrice Mortessagne, Elodie Richalot. Universal intensity statistics in a chaotic reverberation chamber to refine the criterion of statistical field uniformity. Metrology for Aerospace (MetroAeroSpace), 2015 IEEE, IEEE, Jun 2015, Benevento, Italy. pp.225, 10.1109/MetroAeroSpace.2015.7180658 . hal-01203445

\section{HAL Id: hal-01203445 \\ https://hal.science/hal-01203445}

Submitted on 23 Sep 2015

HAL is a multi-disciplinary open access archive for the deposit and dissemination of scientific research documents, whether they are published or not. The documents may come from teaching and research institutions in France or abroad, or from public or private research centers.
L'archive ouverte pluridisciplinaire HAL, est destinée au dépôt et à la diffusion de documents scientifiques de niveau recherche, publiés ou non, émanant des établissements d'enseignement et de recherche français ou étrangers, des laboratoires publics ou privés. 


\section{Universal Intensity Statistics in a Chaotic Reverberation Chamber to Refine the Criterion of Statistical Field Uniformity}

\author{
J.-B. Gros, U. Kuhl, O. Legrand \\ and F. Mortessagne \\ Université Nice-Sophia Antipolis, CNRS, \\ Laboratoire Physique de la Matière Condensée, \\ UMR 7336, 06100 Nice, France, \\ Email: olivier.legrand@unice.fr
}

\author{
E. Richalot \\ Université Paris-Est, ESYCOM (EA 2552), \\ UPEMLV, ESIEE-Paris, CNAM, \\ 77454 Marne-la-Vallée, France
}

\begin{abstract}
This article presents a study of the intensity statistics of the electromagnetic response in a chaotic reverberation chamber (RC) in the presence of losses. Through an experimental investigation, intensity statistics of the response in a conventional mode-stirred $\mathrm{RC}$ are compared with those in a chaotic $\mathrm{RC}$ in the neighborhood of the Lowest Useable Frequency. The present work illustrates how the universal statistical properties of the field in an actual chaotic RC can ensure the validity of the standard criterion proposed to evaluate the uniformity of the field distribution. In particular, through a theoretical approach based on the random matrix theory applied to open chaotic systems, we find that the modal overlap seems to be the only relevant parameter of the corresponding intensity distribution.
\end{abstract}

\section{INTRODUCTION}

Electromagnetic (EM) reverberation chambers are commonly used for electromagnetic compatibility (EMC) tests [1]. Due to mechanical or electronic stirring and to the presence of loss mechanisms leading to modal overlap, the resulting field is assumed to be statistically isotropic, uniform and depolarized. Such properties are well understood and correspond to the so-called Hill's hypotheses [2] when the excitation frequency is well above the lowest useable frequency (LUF) [1]. According to Hill's assumptions [3], in this regime, the EM field can be seen as a random superposition of plane travelling waves. Near the LUF, where generally small or moderate modal overlaps are observed, individual modes will contribute to the EM response. In a conventional $\mathrm{RC}$ with a regular rectangular shape, these low-lying modes display highly non-isotropic patterns yielding a response which cannot be expected to be statistically isotropic and uniform, even in the presence of stirring. To the contrary, these statistical requirements are naturally fulfilled by the vast majority of modes in a chaotic cavity without the help of any stirring process [4]. This generic statistical behavior of modes in a chaotic cavity will be coined ergodicity in the following for the sake of brevity. Numerous studies on EM RCs have considered several cavity shapes of intrinsic chaotic behavior [5]-[7]. By comparing the experimental EM responses in a chaotic $\mathrm{RC}$ and in a conventional mode-stirred RC, the ergodicity of modes in a chaotic RC will be demonstrated to play a key role in improving the statistical behavior of RCs in the neighborhood of the LUF. In particular, we will show that, in a frequency range near the estimated LUF, a generally admitted criterion proposed by the International Standard IEC 610004-21 [1] to evaluate the uniformity of the field distribution is valid in the chaotic RC and not in the conventional one. This important result is related to the universal statistical features of chaotic cavities, in complete contradistinction with what can be obtained in a conventional $\mathrm{RC}$ where the specific details of the chamber lead to unpredictable statistical features due to the lack of ergodicity.

\section{STATISTICS OF THE RESPONSE IN A CHAOTIC RC - THEORETICAL PREDICTIONS}

We briefly give the main features of the statistical behavior of the response of an open chaotic system which verifies the hypotheses introduced by Pnini and Shapiro [8]. Then, using the effective Hamiltonian formalism with the help of random matrix theory applied to open chaotic systems (see for instance [9] and references therein), we will deduce what can be expected for the statistics of the EM response in a chaotic RC [10]. For a given frequency of excitation, the EM response is built upon a sum over resonant modes with mean spacing $\Delta f$ between adjacent resonant frequencies and average width $\Gamma$. From these quantities, one defines the mean modal overlap $d=\Gamma / \Delta f \simeq 8 \pi V f^{3} c^{-3} Q^{-1}$ where $V$ is the volume of the cavity and $Q$ the mean quality factor.

For frequencies much larger than the LUF, generally implying $d \gg 1$, Hill's hypotheses are expected to be valid. The latter yield a complex EM field, each Cartesian component of which having real and imaginary parts which are statistically independent and identically distributed following a normal distribution. In this case, the distribution of the squared modulus of each component follows an exponential law. This regime has been extensively investigated in different contexts such as nuclear physics (Ericson's regime) and room acoustics (Schroeder's regime) [11]. However, near or slightly above the LUF (where $d \lesssim 1$ ), the real and imaginary parts of each component of the field are not identically distributed [10]. For a given frequency of excitation and a given configuration 
of the cavity, in the case of an ideally chaotic RC, they still are independently distributed according to normal laws, but with different variances. Note that the latter statement is true provided the field is appropriately normalized via the transformation $\vec{E} \rightarrow \vec{E} / \sqrt{\int_{V} \vec{E} \cdot \vec{E} d \vec{r}}$ which cancels the global spatial phase of the field [10]. The ensuing distribution of the squared modulus of each component $I_{a}=\left|E_{a}\right|^{2}$ is then no longer exponential and depends on a single parameter $\rho$, called the phase rigidity, defined by:

$$
\rho=\frac{\int_{V} \vec{E} \cdot \vec{E} d \vec{r}}{\int_{V}\|\vec{E}\|^{2} d \vec{r}}
$$

More precisely, in a chaotic $\mathrm{RC}$, due to the ergodicity of the modes contributing to the response, for a given excitation frequency and a given configuration (stirrer position, polarisation and position of the sources), the probability distribution of the normalized intensity of the Cartesian component $\tilde{I}_{a}=\left|E_{a}\right|^{2} /\left\langle\left|E_{a}\right|^{2}\right\rangle_{\vec{r}}$ depends on the sole modulus of $\rho$ and is given by [12]

$$
P\left(\tilde{I}_{a} ; \rho\right)=\frac{1}{\sqrt{1-|\rho|^{2}}} \exp \left[-\frac{\tilde{I}_{a}}{1-|\rho|^{2}}\right] \mathrm{I}_{0}\left[\frac{|\rho| \tilde{I}_{a}}{1-|\rho|^{2}}\right] .
$$

This result was originally proposed by Pnini and Shapiro [8] to model the statistics of scalar fields in partially open chaotic systems. Note that the above distribution continuously interpolates between the two extreme distributions, namely Porter-Thomas for closed systems $(|\rho| \rightarrow 1)$ and exponential for completely open systems $(|\rho|=0)$. Since the phase rigidity is itself a distributed quantity, the distribution of the normalized intensity for an ensemble of responses $\left\{\tilde{I}_{a}\right\}$, obtained for different frequencies or configurations, reads

$$
P_{a}\left(\tilde{I}_{a}\right)=\int_{0}^{1} P_{\rho}(\rho) P\left(\tilde{I}_{a} ; \rho\right) d \rho
$$

where $P_{\rho}$ is the distribution of the phase rigidity of the responses. In the framework of the random matrix theory (RMT) for open chaotic systems, the latter distribution can be evaluated by modeling all types of losses through $M$ channels coupling the inside of the cavity with its environment (including antennas or Ohmic losses at walls). If, for the sake of simplicity, all channels are assumed to be equally coupled, in the limit of weak coupling, the coupling strength denoted $\kappa$ can be shown to be given by $\kappa=\frac{\pi d}{2 M}$. Then, fixing the value of the modal overlap $d$, one can simulate the response of a lossy cavity through the effective Hamiltonian formalism of the scattering matrix [9], [10] with $M$ equally coupled channels of strength given by the above relation.

\section{Statistics OF THE RESPONSE IN A CHAOTIC RC - EXPERIMENTAL REALIZATION}

This section presents experimental results that were obtained in a commercial RC equipped with a vertical stirrer. The same RC was rendered chaotic by the addition of 3 metallic half-spheres on three adjacent walls (cf Fig. 1). The chaotic character of such a modified RC was verified using the methods described in [4].

In both configurations of this RC (bare or with halfspheres) $\left(V \simeq 19 \mathrm{~m}^{3}\right)$, the $S$-matrix was measured between two

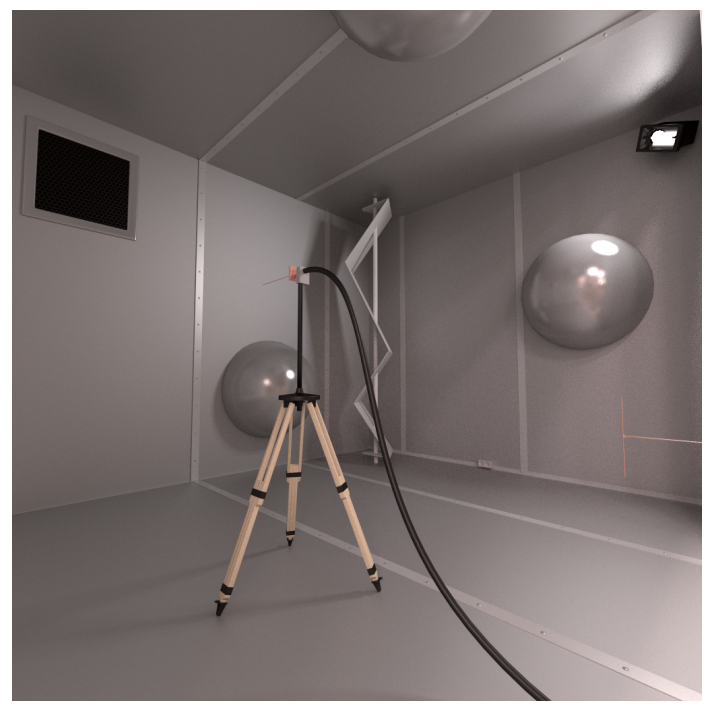

Fig. 1. Artist view of the reverberation chamber made chaotic through the addition of 3 half-spheres. The volume of the RC is $19.1 \mathrm{~m}^{3}$ without the halfspheres, each having a radius of $40 \mathrm{~cm}$. A vertical stirrer can be seen in the far corner and a monopole antenna fixed on a wooden tripod is shown.

antennas (one dipole fixed at a wall and one monopole fixed on a moveable tripod) located at $\vec{r}_{1}$ and $\vec{r}_{2}$. Measurements were realized for 1024 regularly spaced frequencies in the interval $[309 \mathrm{MHz}, 410 \mathrm{MHz}$ ], for 30 positions of the stirrer spaced by 12 degrees and for 8 different positions of the monopole antenna inside the volume. After extracting the coupling strength of the antennas [9], [10], one can deduce from the measurement of $S_{12}$ the normalized value of the amplitude of the Cartesian component of the field along the monopole antenna:

$$
\vec{E}\left(\vec{r}_{2}, f\right) \cdot \hat{n}_{a}=\frac{S_{12}(f)}{\kappa_{1} \kappa_{2}} .
$$

where $\kappa_{i}(i=1,2)$ are the stirring-averaged values of the frequency-dependent coupling constants of the antennas, and $\hat{n}_{a}$ is the unit vector along the polarisation of antenna 2 . In the frequency range of our study (of the order of 7 times the cut-off frequency), the mean quality factor was estimated to be around 1500 (almost insensitive to the presence of the half-spheres) corresponding to a mean modal overlap $d \sim 0.89$, which was deduced directly from the measurements by extracting the complex resonances using the method of harmonic inversion [13]. From the measured value of $d$, we performed numerical random matrix computations to deduce the corresponding numerical distribution of the phase rigidity which we inserted in expression (3) for the normalized intensity distribution.

In Fig. 2, the empirical histograms of the normalized intensities of the field components (for all the excitation frequencies and all configurations) for both RCs are compared with the prediction (3). To evaluate the latter, the distribution $P_{\rho}$ is deduced from a RMT numerical computation with $d=0.89$ (see inset). The excellent agreement between expression (3) and the empirical distribution associated to the chaotic RC (red histogram) clearly demonstrates that the assumption of ergodicity holds in the chaotic RC. These results also confirm that the modal overlap seems to be the only relevant parameter of the corresponding intensity distribution. Note that none of 


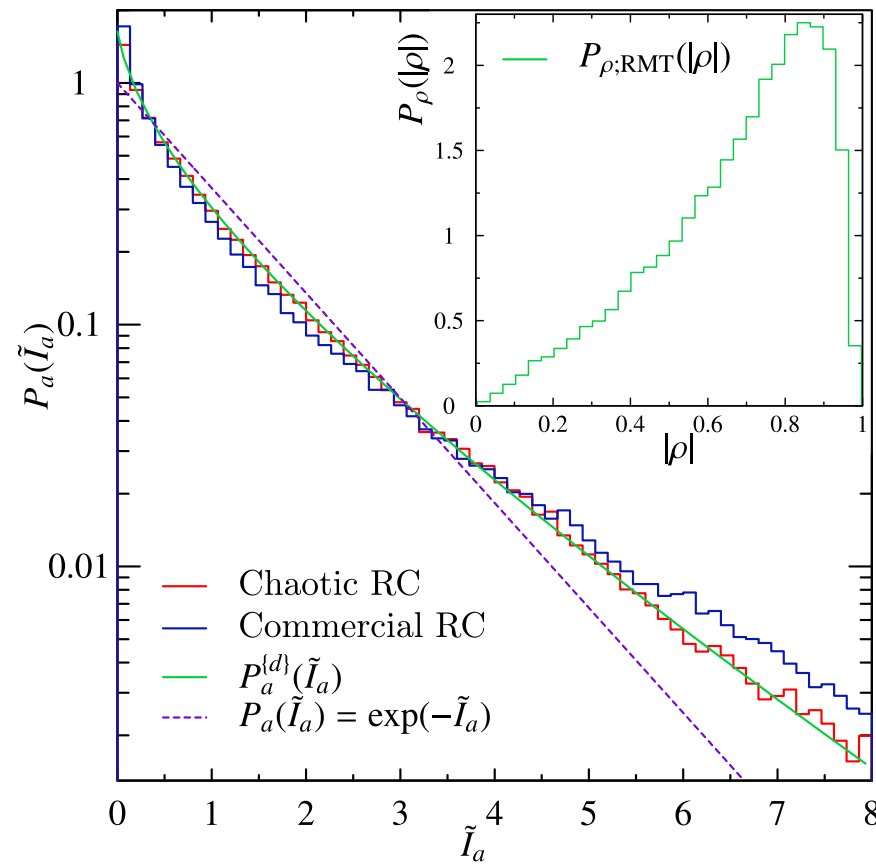

Fig. 2. Empirical distributions of the normalized intensities: blue histogram for the conventional RC and red histogram for the chaotic RC. Comparison with the prediction (3) (green continuous curve) where $P_{\rho}$ is the distribution deduced from a random matrix computation with $d=0.89$ (see inset). The dashed purple curve shows the exponential distribution expected under Hill's hypotheses.

the empirical distributions agree with the exponential distribution (dashed purple curve) which is expected when Hill's hypotheses hold.

Preliminary investigations, based on numerical simulations of the RMT type mentioned in the previous section [10], show that $P_{\rho}$ only depends on $d$, irrespective of the values of $M$ and $\kappa$, as long as the coupling and the modal overlap are weak or moderate. This single parameter dependence can be substantiated through a simplified analytical approach based on $2 \times 2$ random matrices where equal widths are assumed. Then, using the Wigner surmise for the spacing distribution, one can show that $P_{\rho}$ has the analytical form:

$$
P_{\rho}^{W}(\rho)=\frac{2 B \exp [-2 B \rho /(1-\rho)]}{(1-\rho)^{2}},
$$

where $B$ should be considered as a free parameter whose limiting value would be $\pi d^{2} / 4$ in the weak overlap limit [14]. To check this surmise concerning the distribution of the phase rigidity of the responses, we investigated a statistical ensemble of responses numerically evaluated via a sum of resonances taken from $1000 \times 1000$ random matrices of the Gaussian Orthogonal Ensemble (GOE) (see for instance [9]) with all widths set to a single value $\Gamma$ such that $d=\Gamma / \Delta f$. For different values of $d$ ranging from 0.15 to 1 , we fitted the resulting numerical distributions of the phase rigidity with expression (5), from which we could deduce an overall smooth monotonic $d$-dependence of $B$ of the type :

$$
B(d)=\frac{a d^{2}}{1+b d} .
$$

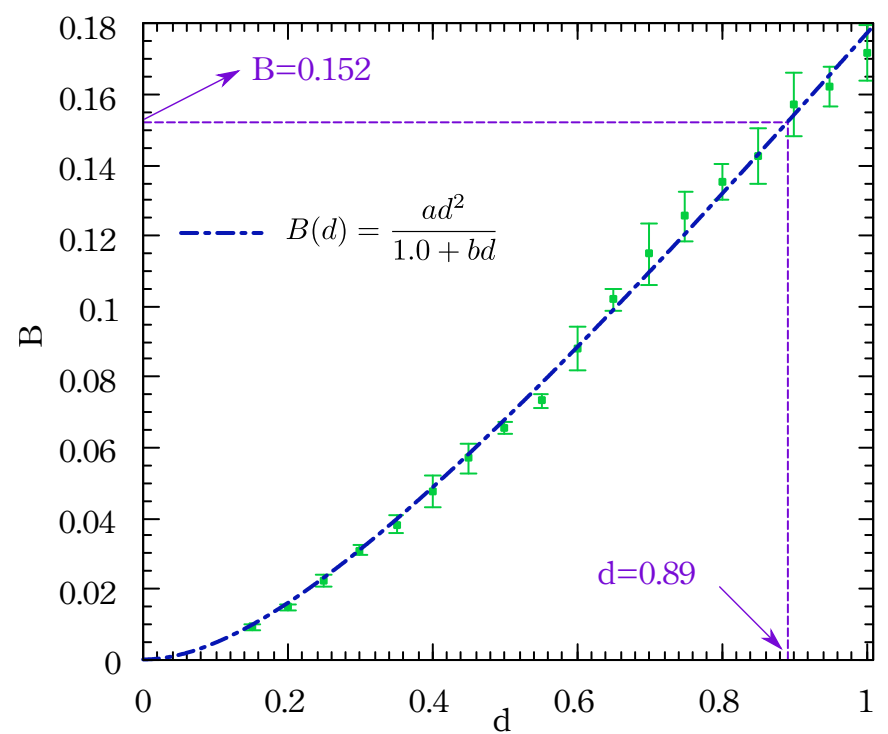

Fig. 3. Empirical evaluation of $B$ parameter as a function of $d$ obtained by fitting an ensemble of distributions of phase rigidity (deduced from statistical ensembles of responses numerically evaluated via a sum of resonances taken from GOE random matrices where all widths are set to a single value $\Gamma$ such that $d=\Gamma / \Delta f$ ) with the ansatz (5). The dashed-dotted curve is obtained through a fit of $B$ with expression (6), yielding $a=0.58 \pm 0.04$ and $b=$ $2.3 \pm 0.3$. The value of $B=0.152$ corresponding to the experimental value of $d=0.89$ is indicated.

This behavior is illustrated in Fig. 3. To be more specific, for each value of $d$, we performed 10 different numerical simulations each yielding one value for $B$. For each numerical simulation, 50 GOE random matrices of rank 1000 were generated, from which 101 independent responses were evaluated in the vicinity of the center of the spectrum. From these $50 \times 101$ responses, a histogram of the phase rigidity was obtained which was then fitted by expression (5). Thus, for a given value of $d, 10$ values of $B$ were estimated (yielding the shown error bars). The dashed-dotted curve is finally obtained through a fit of $B$ with the above expression (6), yielding $a=0.58 \pm 0.04$ and $b=2.3 \pm 0.3$.

To fix ideas about the relevance of the ansatz (5), a typical plot of its expression is given in Fig. 4 for a value of $B=0.152$ which is associated to the value of $d=0.89$ deduced from experimental results. It bears a clear resemblance to the histogram shown as inset of Fig. 2 in spite of differences for small values of $\rho$ which are most likely due to the oversimplified approach leading to the ansatz (5).

We naturally wished to test the validity of the above ansatz (5) proposed for the distribution of the phase rigidity, and more specifically the dependence of the $B$-parameter as a function of the modal overlap $d$ shown in Fig. 3. To this end, we compared the empirical distribution of the normalized intensities with prediction (3) where we used expression (5) and the value of $B=0.152$ evaluated through equation (6) with the measured value of $d=0.89$ (see Fig. 3). The resulting analytical prediction is in good agreement with the empirical one and is nearly indistinguishable from the previously mentioned RMT prediction (green curve of Fig. 2). In order to compare these two predictions, we computed their root-mean-square errors with respect to the empirical distribution. Both are very close. 


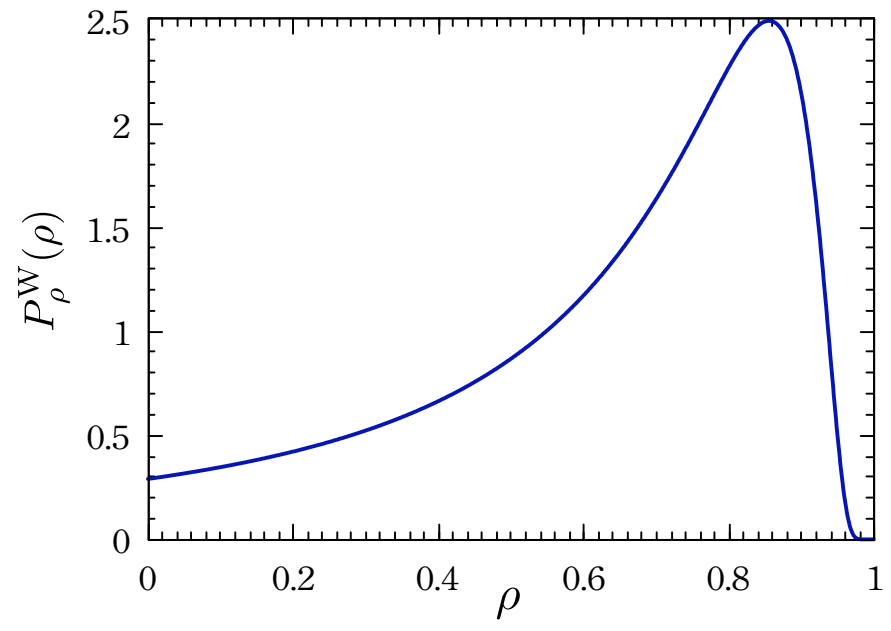

Fig. 4. A plot of expression (5) with $B=0.152$ which is associated to the measured value of $d=0.89$ through the expression (6) shown in Fig. 3.

Indeed, the root-mean-square error is around 0.019 for the prediction using expression (5) whereas it is around 0.013 with the RMT prediction. This agreement strongly substantiates our guess that he distribution of the phase rigidity seems to depend only on the modal overlap, at least in the range of parameters that we could explore, which corresponds to a regime of weak coupling and moderate modal overlap. We suggest that the approach described above could provide an alternative cost-effective mean of extracting the modal overlap parameter $d$, within a given frequency range, only by inspecting spatial statistics (e.g. through the distribution of the normalized intensity) and without the need of a frequency analysis involving the method of harmonic inversion which may prove to be not so straightforward when the modal overlap becomes of the order of unity.

We finally wish to test how the above results can help understand whether the field uniformity proposed by the International Standard IEC 61000-4-21 [1] is still relevant near the LUF. According to the latter, the fluctuations of the maxima of the field amplitude can be evaluated through [1]:

$$
\left.\sigma_{d B}(f)=20 \log _{10}\left(1+\frac{\sigma_{\max }}{\left\langle\left|E_{a}\right| \max \right.}\right\rangle\right)
$$

where 8 measurement points are chosen in the RC distant from at least a quarter of a wavelength. At each of these points, for 30 uncorrelated positions of the stirrer, one extracts the amplitude of the field $\left|E_{a}\right|$ and one keeps the maximum value $\left|E_{a}\right|_{\max }$. One then computes the average and the standard deviation over the 8 values of $\left|E_{a}\right|_{\max }$. According to [1], the field can be considered as uniform when $\sigma_{d B}<3 \mathrm{~dB}$. In Fig. 5 we present a comparison of histograms of $\sigma_{d B}$ obtained experimentally in the two configurations mentioned above.The chaotic RC (red curve of Fig. 5) complies almost always with this criterion (only $6 \%$ above $3 \mathrm{~dB}$ ) and in a much better way than the conventional RC (blue dashed curve of Fig. 5) does (about $30 \%$ above $3 \mathrm{~dB}$ ). To correctly interpret these findings, one should first note that, according to numerical simulations of the EM responses in both $\mathrm{RCs}$ which are not presented here, the frequencies for which $\sigma_{d B}$ is larger than $3 \mathrm{~dB}$, almost always correspond to EM response patterns which are clearly

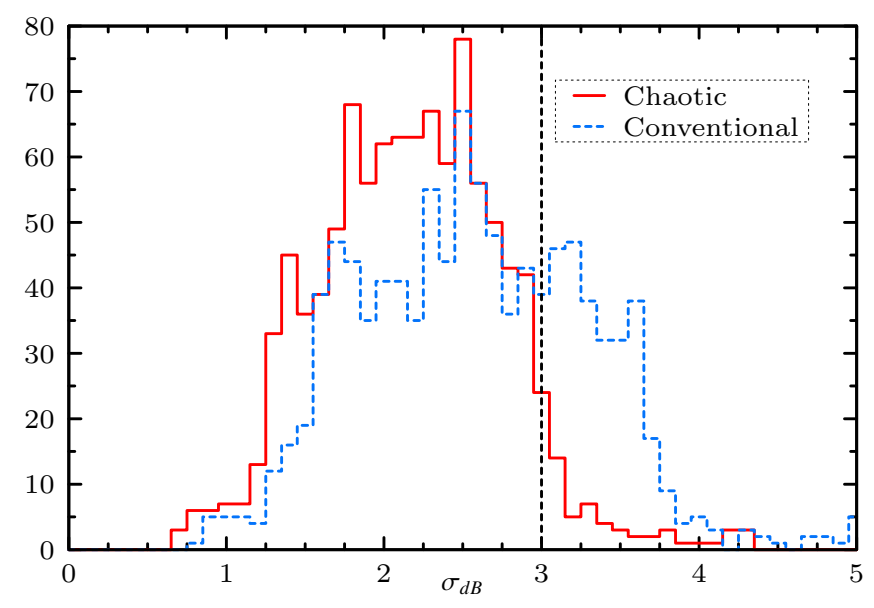

Fig. 5. Fluctuations of the measured field amplitude maxima through histograms of $\sigma_{d B}$ defined by (7). Chaotic RC: red continuous histogram. Conventional RC: blue dashed histogram. The chaotic RC displays only $6 \%$ above $3 \mathrm{~dB}$, whereas $30 \%$ lie above $3 \mathrm{~dB}$ in the conventional RC

not ergodic. Moreover, by increasing the number of points of measurements, one numerically observes that the histograms of $\sigma_{d B}$ tend to concentrate around a unique average value well below $3 \mathrm{~dB}$ in the chaotic RC whereas such a concentration is not observed in the conventional RC. Again, the deep reason for this difference stems upon the ergodicity of the response in the chaotic RC which yields universal statistics which can be fully accounted for through the universal properties of the random matrix theory applied to open chaotic wave systems.

Hence, the above comparisons between chaotic and conventional RCs demonstrate that ergodicity ensuing chaoticity is the only certain way of obtaining meaningful results when resorting to the uniformity criterion introduced by the standard IEC 61000-4-21 to perform EMC tests.

\section{CONCLUSION}

This article presents an experimental investigation of the statistics of the EM response in a mode-stirred reverberation chamber made chaotic by adding spherical elements on its walls. A theoretical approach based on the random matrix theory applied to chaotic open wave systems enables us to predict the distribution of the normalized intensity of the response in terms of a unique parameter, namely the mean modal overlap. By means of a simplified random matrix approach, we propose an ansatz expression for the distribution of the phase rigidity of the response, with a unique free parameter which we can numerically show to exhibit a simple smooth monotonic dependence on the mean modal overlap. This theoretical prediction is successfully compared to our measurements in a chaotic RC, thereby confirming the key role of the ergodic character of the response of a chaotic $\mathrm{RC}$ to improve the statistical behavior of an RC in the neighborhood of the LUF. In particular, these results demonstrate that the criterion proposed by the standard to evaluate the uniformity of the spatial field distribution, when used in the vicinity of the LUF and in a regime of moderate modal overlap, is only relevant if the $\mathrm{RC}$ is chaotic since the ergodicity of the response can only be guaranteed in that case. 


\section{ACKNOWLEDGMENT}

The authors acknowledge financial support by the French National Research Agency (ANR) via the project CAOREV.

\section{REFERENCES}

[1] CISPR/A and IEC SC 77B, IEC 61000-4-21,"Electromagnetic Compatibility (EMC)- Part 4-21: Testing and Measurement Techniques Reverberation Chamber Test Methods", International Electrotechnical Commission (IEC) International Standard.

[2] D. Hill, Electromagnetic Fields in Cavities: Deterministic and Statistical Theories, IEEE Press Series on Electromagnetic Wave Theory, IEEE; Wiley, 2009.

[3] D. Hill, "Plane wave integral representation for fields in reverberation chambers", IEEE Trans. Electromagn. Compat. 40: 209, 1998.

[4] J.-B. Gros, O. Legrand, F. Mortessagne, E. Richalot, K. Selemani, "Universal behaviour of a wave chaos based electromagnetic reverberation chamber", Wave Motion 51: 664, 2014.

[5] U. Dörr, H.-J. Stöckmann, M. Barth, U. Kuhl, "Scarred and chaotic field distributions in a three-dimensional Sinai-microwave resonator, Phys. Rev. Lett. 80 : 1030, 1998.

[6] V. Galdi, I. M. Pinto, and L. B. Felsen, "Wave propagation in raychaotic enclosures: Paradigms, oddities and examples, IEEE Antennas Propag. Mag. 47: 612626, 2005.

[7] G. Orjubin, E. Richalot, O. Picon, and O. Legrand, "Wave chaos techniques to analyze a modeled reverberation chamber, Comptes Rendus Physique $10: 42,2009$.

[8] R. Pnini and B. Shapiro, "Intensity fluctuations in closed and open systems", Phys. Rev. E, 54: R1032, 1996.

[9] U. Kuhl, O. Legrand, F. Mortessagne, "Microwave experiments using open chaotic cavities in the realm of the effective Hamiltonian formalism", Fortschritte der Physik / Progress of Physics 61: 404, 2013.

[10] J.-B. Gros, "Statistiques spatiales des cavités chaotiques ouvertes: Applications aux cavités chaotiques électromagnétiques", PhD thesis, 2014.

[11] O. Legrand, F. Mortessagne, D. Sornette, "Spectral Rigidity in the Large Modal Overlap Regime: Beyond the Ericson-Schroeder Hypothesis", Journal de Physique I, 5: 1003, 1995. Erratum: Ibid 5: 1517, 1995.

[12] Y.-H. Kim, U. Kuhl, H.-J. Stöckmann, P. Brouwer, "Measurement of Long-Range Wave-Function Correlations in an Open Microwave Billiard", Phys. Rev. Lett., 94: 036804, 2005.

[13] J.-B. Gros, U. Kuhl, O. Legrand, F. Mortessagne, E. Richalot, D. V. Savin, "Experimental width shift distribution: a test of nonorthogonality for local and global perturbations", Phys. Rev. Lett.,113: 224101, 2014

[14] J.-B. Gros, U. Kuhl, O. Legrand, F. Mortessagne, in preparation. 